Research

\title{
Assessment of tissue oxygen saturation during a vascular occlusion test using near-infrared spectroscopy: the role of probe spacing and measurement site studied in healthy volunteers
}

\author{
Rick Bezemer ${ }^{1,2}$, Alexandre Lima ${ }^{1}$, Dean Myers ${ }^{3}$, Eva Klijn ${ }^{1}$, Michal Heger ${ }^{4}$, Peter T Goedhart ${ }^{2}$, \\ Jan Bakker ${ }^{1}$ and Can Ince ${ }^{1,2}$

\begin{abstract}
1'Department of Intensive Care, Erasmus MC, University Medical Center Rotterdam, 's-Gravendijkwal 230, 3015 CE Rotterdam, the Netherlands 2Department of Translational Physiology, Academic Medical Center, University of Amsterdam, Meibergdreef 9, 1105 AZ Amsterdam, the Netherlands ${ }^{3}$ Hutchinson Technology Inc., 40 West Highland Park Drive NE, Hutchinson, MN 55350-9784, USA

${ }^{4}$ Department of Experimental Surgery, Academic Medical Center, University of Amsterdam, Meibergdreef 9, 1105 AZ Amsterdam, the Netherlands
\end{abstract}

Corresponding author: Rick Bezemer, r.bezemer@amc.uva.nl

Published: 30 November 2009

This article is online at http://ccforum.com/content/13/S5/S4

(c) 2009 BioMed Central Ltd
Critical Care 2009, 13(Suppl 5):S4 (doi:10.1186/cc8002)

$\mathrm{StO}_{2}$ parameters of the hyperemic phase of the VOT, such as the area under the curve, significantly correlated to the minimum $\mathrm{StO}_{2}$ during ischemia.

Conclusions: NIRS measurements in combination with a VOT are measurement site-dependent and probe-dependent. Whether this dependence is anatomy-, physiology-, or perhaps technologyrelated remains to be elucidated. Our study also indicated that reactive hyperemia depends on the extent of ischemic insult.

\section{Introduction}

It is now well established that tissue oxygen utilization and regional microcirculatory oxygen transport properties are severely affected during sepsis and shock [1-9]. To assess and identify these metabolic and microcirculatory alterations non-invasively, near-infrared spectroscopy (NIRS) has recently been applied to measure the behavior of tissue oxygen saturation $\left(\mathrm{StO}_{2}\right)$. Besides observation of steadystate values, a vascular occlusion test (VOT) has been introduced for the measurement of tissue oxygen consumption and of microvascular reperfusion and reactivity [9-14].

The VOT seems to be sensitive to the progress and outcome of sepsis in critical illness $[9,12]$. A large number of variables exist in the performance of this maneuver, however, yielding apparently conflicting results and uncertainty as to the significance of the various VOT-derived $\mathrm{StO}_{2}$ parameters [11]. The analysis, interpretation, and understanding of VOTderived $\mathrm{StO}_{2}$ traces, although being widely employed in septic and trauma patients, is limited, especially for the postocclusion phase of the VOT. Consequently, identification of

$\mathrm{NIRS}=$ near-infrared spectroscopy $; \mathrm{StO}_{2}=$ tissue oxygen saturation; $\mathrm{VOT}=$ vascular occlusion test. 
which $\mathrm{StO}_{2}$ parameters are most appropriate for scoring (micro)vascular reperfusion and reactivity remains to be determined. Proper characterization of VOT-derived $\mathrm{StO}_{2}$ parameters in health is hence needed to allow translation of results obtained in patients to pathophysiological phenomena.

The main problem with the interpretation of $\mathrm{StO}_{2}$ data in the literature is the diversity of methodologies used for assessing $\mathrm{StO}_{2}$ during a VOT. Results vary from study to study, making data comparison and interpretation difficult and possibly inadequate. Two major aspects regarding the inconsistent methodology are the measurement site and probe spacing (that is, the spatial separation between the illumination and detection fibers of the NIRS probe). The measurement site is important because differences may exist in the sensitivity of muscle groups and/or other anatomical structures to the VOT during health and/or pathophysiological conditions. Probe spacing, on the other hand, will determine the depth of measurement within the respective muscle group. To study the roles of both variables, we performed 3-minute VOTs in healthy volunteers and measured $\mathrm{StO}_{2}$ using $15 \mathrm{~mm}$ and $25 \mathrm{~mm}$ probe spacings on the thenar and the forearm. VOTderived $\mathrm{StO}_{2}$ traces were quantified for baseline, ischemic, reperfusion, and hyperemic $\mathrm{StO}_{2}$ parameters. We expect these results to provide an essential frame of reference for conducting $\mathrm{StO}_{2}$ measurements in future clinical studies.

\section{Materials and methods Subjects}

The study protocol was approved by the Medical Ethics Committee of the Erasmus Medical Center Rotterdam. Eight healthy volunteers (Table 1) who were not receiving any vasoactive medication were requested to refrain from consuming caffeine-containing beverages prior to the experiments. The subjects were comfortably seated in the experimental room (mean \pm standard deviation room temperature was $21 \pm 1^{\circ} \mathrm{C}$ ) 1 hour before measurements and were requested not to perform any physical labor (for example, lifting and writing).

\section{Near-infrared spectroscopy}

$\mathrm{StO}_{2}$ was continuously and non-invasively measured using two InSpectra tissue spectrometers (Model 325; Hutchinson Technology, Hutchinson, MN, USA). The spectrometers use reflectance mode probes that have a $1.5 \mathrm{~mm}$ optical fiber to illuminate the tissue and a $0.4 \mathrm{~mm}$ optical fiber to collect the backscattered light from the tissue. Two types of probes were used for this study: one with $15 \mathrm{~mm}$ spacing between the illumination and the collecting optical fibers, and one with $25 \mathrm{~mm}$ spacing. Both probes have been used in various studies with varying results [9-12].

The relative optical attenuation of the backscattered light at four wavelengths $(680 \mathrm{~nm}, 720 \mathrm{~nm}, 760 \mathrm{~nm}$, and $800 \mathrm{~nm})$ is measured to calculate two second-derivative attenuation values, one centered at $720 \mathrm{~nm}$ and the other at $760 \mathrm{~nm}$ [15]. The ratio of the $720 \mathrm{~nm}$ to $760 \mathrm{~nm}$ second-derivative
Table 1

\begin{tabular}{lc} 
Demographic characteristics of the studied subjects \\
\hline Demographic characteristic & Mean \pm standard deviation \\
\hline Age (years) & $28 \pm 6$ \\
Gender (\%) & 25 \\
$\quad$ Female & 75 \\
$\quad$ Male & \\
Dominant hand (\%) & 87.5 \\
$\quad$ Right & 12.5 \\
$\quad$ Left & $78 \pm 10$ \\
Weight (kg) & $178 \pm 8$ \\
Height (cm) & $36.6 \pm 0.5$ \\
Tympanic temperature $\left({ }^{\circ} \mathrm{C}\right)$ & $112 \pm 9$ \\
Systolic blood pressure $(\mathrm{mmHg})$ & $73 \pm 7$ \\
Diastolic blood pressure $(\mathrm{mmHg})$ & $91 \pm 8$ \\
Mean arterial pressure $(\mathrm{mmHg})$ & $69 \pm 8$ \\
Heart rate (beats/minute) & \\
\hline
\end{tabular}

values is directly extrapolated to $\mathrm{StO}_{2}$, defined as $\left[\mathrm{HbO}_{2}\right] /$ $\left([\mathrm{Hb}]+\left[\mathrm{HbO}_{2}\right]\right)$, via a calibration table. The calibration table relating $\mathrm{StO}_{2}$ to the second-derivative attenuation ratio is stored permanently within the NIRS device and is common to each device and probe used [15]. The NIRS devices were calibrated before the first measurement in each subject using a light-scatter calibrator.

\section{Vascular occlusion test}

One NIRS probe was placed on the skin of the thenar eminence and another NIRS probe was placed on the lateral side of the anterior surface of the forearm for simultaneous measurement of thenar $\mathrm{StO}_{2}$ and forearm $\mathrm{StO}_{2}$ during the VOTs. Both the hand and the forearm were kept at heart level with the palms up. The subjects were instructed not to move their hand or arm, or to change their sitting position during measurements.

Baseline arterial pressure was measured using a manual sphygmomanometer. After a 3-minute stabilization period (baseline measurement), stagnant ischemia was induced for 3 minutes by rapidly inflating a pneumatic cuff (within 5 seconds), placed around the upper arm, to $50 \mathrm{mmHg}$ above systolic blood pressure. The cuff was subsequently deflated (within 1 second) and $\mathrm{StO}_{2}$ measurements were continued up to 5 minutes post ischemia.

\section{$\mathrm{StO}_{2}$ curve characteristics}

$\mathrm{StO}_{2}$ data from the two devices were continuously saved (one sample every 3.5 seconds) on two computers and were 
retrospectively analyzed using InSpectra Analysis V3.3 software (Hutchinson Technology). The VOT-derived $\mathrm{StO}_{2}$ traces were divided into four phases: baseline, ischemia, reperfusion, and hyperemia (Figure 1).

The ischemic phase was analyzed for $\mathrm{StO}_{2}$ downslope (\%/minute), minimum $\mathrm{StO}_{2}$ after 3 minutes of ischemia (\%), and $\Delta \mathrm{StO}_{2}(\%$; that is, the difference between baseline and minimum $\mathrm{StO}_{2}$ ). The $\mathrm{StO}_{2}$ downslope is generally considered to reflect muscle metabolism and the minimum $\mathrm{StO}_{2}$ is considered to indicate the extent of ischemia.

The reperfusion phase was analyzed for two parameters: $\mathrm{StO}_{2}$ upslope (\%/minute) and rise time (minutes), both measured over the interval from minimum $\mathrm{StO}_{2}$ to baseline $\mathrm{StO}_{2}$ (Figure 1). Although these parameters are directly related $\left(\mathrm{StO}_{2}\right.$ upslope $=\Delta \mathrm{StO}_{2} /$ Rise time), the $\mathrm{StO}_{2}$ upslope is metabolism-dependent as it is based on $\Delta \mathrm{StO}_{2}$ after a fixed time of occlusion (that is, 3 minutes), while the rise time solely represents the time required to wash out the stagnantly deoxygenated blood by oxygenated arterial blood during reperfusion. These two parameters were therefore measured and analyzed separately.

The hyperemic phase of the VOT was analyzed for peak $\mathrm{StO}_{2}$ during reperfusion (\%), for $\mathrm{StO}_{2}$ overshoot (that is, difference between peak $\mathrm{StO}_{2}$ and baseline $\mathrm{StO}_{2}$ ), for the area under the hyperemic curve (AUC; \%-minute), and for the settling time from release of the cuff to recovery to baseline $\mathrm{StO}_{2}$ (minutes).

\section{Measurement protocol}

Three measurement variables were investigated and compared for the assessment of VOT-derived $\mathrm{StO}_{2}$ parameters: dominant arm versus nondominant arm, forearm versus thenar, and superficial tissue versus deep tissue (as measured by the different probe spacings). For this purpose, four measurements were performed per subject: two on the dominant side and two on the nondominant side. Although good reproducibility of NIRS measurements during sequential VOTs has been demonstrated by Gómez and colleagues [11], the side and probes were switched after every VOT. Additionally, to avoid any effect of starting conditions, the first measurement in four subjects was performed on the dominant side with the $15 \mathrm{~mm}$ probe on the forearm and the $25 \mathrm{~mm}$ probe on the thenar, whereas in the other four subjects the first measurement was performed on the nondominant side with the $15 \mathrm{~mm}$ probe on the thenar and the $25 \mathrm{~mm}$ probe on the forearm.

\section{Statistical analysis}

First, differences between the dominant arm and the nondominant arm were analyzed and data were subsequently categorized into four groups: $15 \mathrm{~mm}$ probe on the forearm $\left(F_{15 \mathrm{~mm}}\right), 25 \mathrm{~mm}$ probe on the forearm $\left(F_{25 \mathrm{~mm}}\right), 15 \mathrm{~mm}$ probe on the thenar $\left(T_{15 \mathrm{~mm}}\right)$, and $25 \mathrm{~mm}$ probe on the thenar
Figure 1

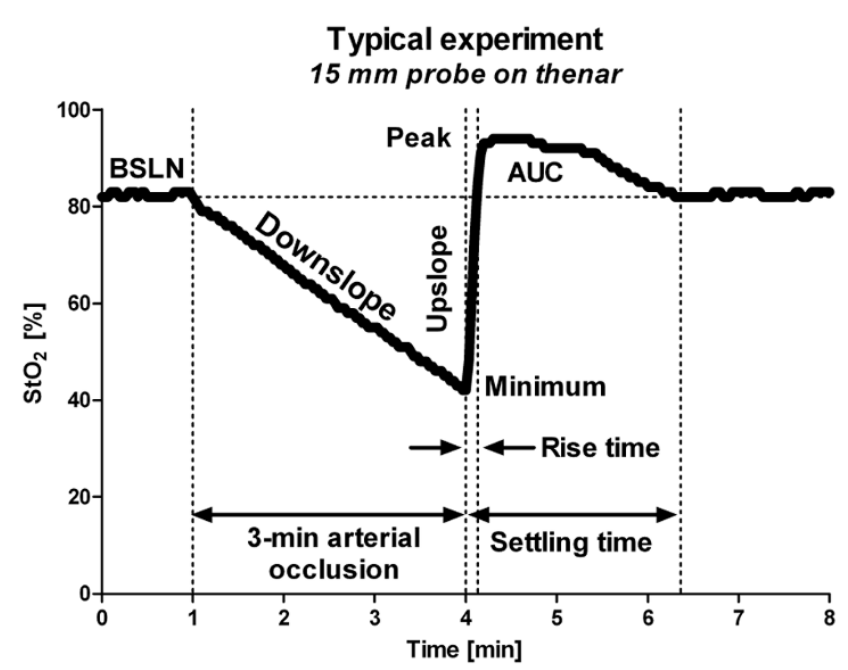

Vascular occlusion test-derived tissue oxygen saturation phases and parameters. The vascular occlusion test-derived tissue oxygen saturation $\left(\mathrm{StO}_{2}\right)$ traces were divided into four phases (baseline, ischemia, reperfusion, and hyperemia) and were analyzed for baseline $\mathrm{StO}_{2}$ (BSLN), $\mathrm{StO}_{2}$ downslope, minimum $\mathrm{StO}_{2}, \mathrm{StO}_{2}$ upslope, rise time, peak $\mathrm{StO}_{2}$, area under the hyperemic curve (AUC), and settling time.

$\left(T_{25 \mathrm{~mm}}\right)$. Statistical analysis was performed in GraphPad Prism software (GraphPad Software, San Diego, CA, USA). Normal distribution of the data within all groups (dominant, nondominant, $F_{15 \mathrm{~mm}}, F_{25 \mathrm{~mm}}, T_{15 \mathrm{~mm}}$, and $T_{25 \mathrm{~mm}}$ ) was confirmed for each $\mathrm{StO}_{2}$ parameter using the D'Agostino and Pearson omnibus normality test. Comparative analysis between groups was performed using analysis of variance with a Bonferroni post-hoc test. Correlation analysis was performed by Pearson's analysis for normally distributed datasets. All data are presented as the mean \pm standard deviation. Differences between groups with $P<0.05$ were considered statistically significant.

\section{Results}

No differences between the dominant and nondominant sides were found and data could therefore be categorized into four groups: $F_{15 \mathrm{~mm}}, \mathrm{~F}_{25 \mathrm{~mm}}, \mathrm{~T}_{15 \mathrm{~mm}}$, and $\mathrm{T}_{25 \mathrm{~mm}}$. Baseline $\mathrm{StO}_{2}$ was similar in all groups and independent of probe spacing and measurement site: $81 \pm 10 \%$ for $F_{15 \mathrm{~mm}}, 85 \pm 7 \%$ for $F_{25 \mathrm{~mm}}$, $87 \pm 4 \%$ for $T_{15 \mathrm{~mm}}$, and $87 \pm 3 \%$ for $T_{25 \mathrm{~mm}}$. Occlusion of the upper arm by a pneumatic cuff resulted in an immediate decrease in $\mathrm{StO}_{2}$. Release of the occlusion after 3 minutes was followed by a rapid increase in $\mathrm{StO}_{2}$ and a $\mathrm{StO}_{2}$ overshoot relative to baseline. Less than 3 minutes postischemia, $\mathrm{StO}_{2}$ values were restored to baseline level.

\section{Ischemic phase}

The downslopes during ischemia were measured using linear regression analysis over the linear part (that is, $R^{2}>0.95$ ) of 

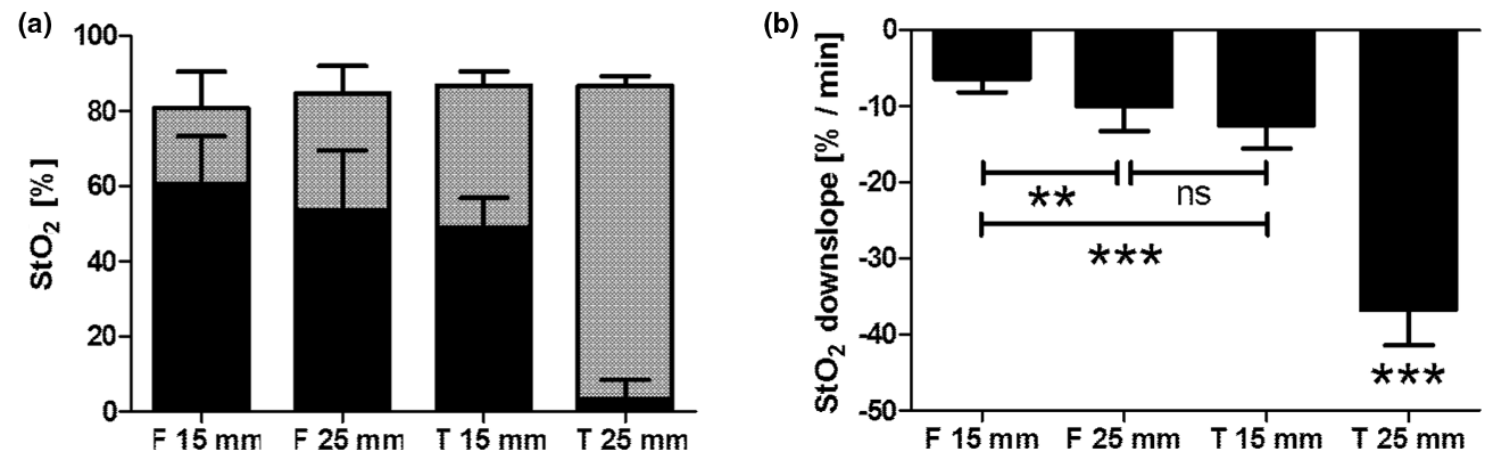

Tissue oxygen saturation at baseline and after ischemia, and the corresponding downslopes. (a) Measured tissue oxygen saturation $\left(\mathrm{StO}_{2}\right)$ baseline values and minima after 3 minutes of ischemia. (b) Corresponding $\mathrm{StO}_{2}$ downslopes (right). ns $=$ not significant $(P>0.05)$, ${ }^{\star \star} P<0.01$, ${ }^{\star \star \star} P<0.001$. F, forearm; $T$, thenar.

Figure 3
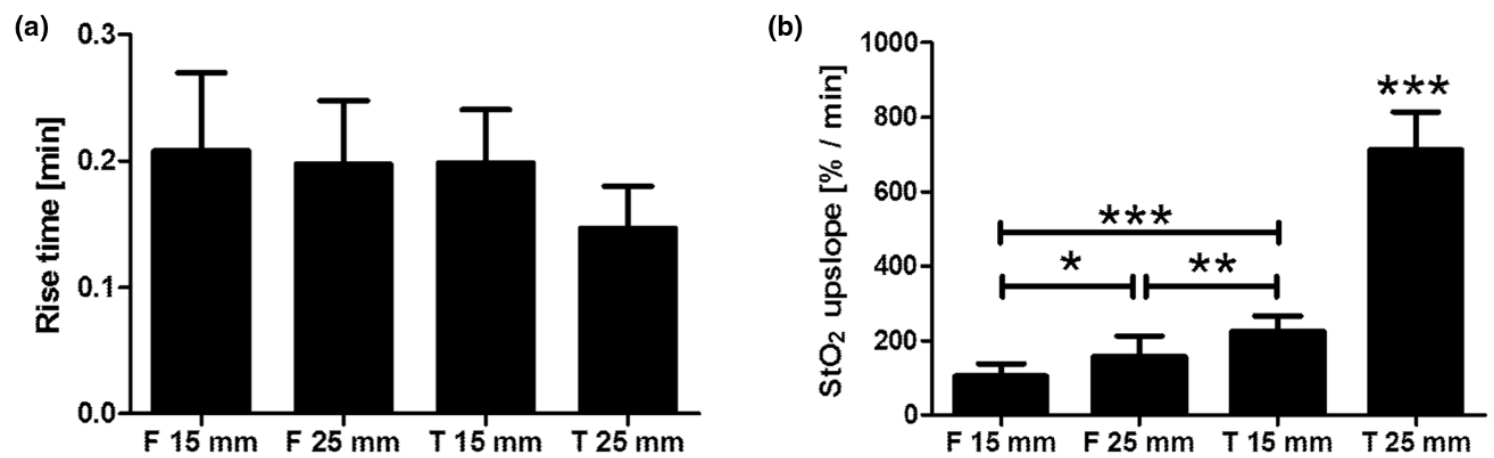

Measured rise times and the corresponding tissue oxygen saturation upslopes. (a) Measured rise times. (b) Corresponding tissue oxygen saturation $\left(\mathrm{StO}_{2}\right)$ upslopes. ${ }^{\star} P<0.05,{ }^{\star \star} P<0.01,{ }^{\star \star \star} P<0.001 . \mathrm{F}$, forearm; $\mathrm{T}$, thenar.

the $\mathrm{StO}_{2}$ curve. For the first three groups $\left(\mathrm{F}_{15 \mathrm{~mm}}, \mathrm{~F}_{25 \mathrm{~mm}}\right.$, and $\mathrm{T}_{15 \mathrm{~mm}}$ ), the downslopes were linear over the entire 3-minute period of ischemia. The downslopes measured in the $T_{25 \mathrm{~mm}}$ group, in contrast, were linear over a time interval of $2.34 \pm$ 0.38 minutes. For $F_{15 \mathrm{~mm}}, \mathrm{~F}_{25 \mathrm{~mm}}, \mathrm{~T}_{15 \mathrm{~mm}}$, and $\mathrm{T}_{25 \mathrm{~mm}}, \mathrm{StO}_{2}$ downslopes were $-6.4 \pm 1.7 \% /$ minute, $-10.0 \pm 3.2 \% /$ minute, $-12.5 \pm 3.0 \% /$ minute, and $-36.7 \pm 4.6 \% /$ minute, respectively (Figure 2). Hence, during 3 minutes of ischemia, $\mathrm{StO}_{2}$ decreased significantly $(P<0.001)$ by $20 \pm 5 \%$, $31 \pm 11 \%$, $38 \pm 9 \%$, and $84 \pm 6 \%$ for $F_{15 \mathrm{~mm}}, F_{25 \mathrm{~mm}}, \mathrm{~T}_{15 \mathrm{~mm}}$, and $\mathrm{T}_{25 \mathrm{~mm}}$, respectively, which resulted in a minimum $\mathrm{StO}_{2}$ of $60 \pm 13 \%$, $54 \pm 16 \%, 49 \pm 8 \%$, and $3 \pm 5 \%$ in these groups (Figure 2).

For all groups, minimum $\mathrm{StO}_{2}$ values were significantly lower than baseline values $(P<0.001)$. For the downslopes and $\Delta \mathrm{StO}_{2}$ during ischemia, values for $\mathrm{F}_{15 \mathrm{~mm}}$ differed significantly from values for $\mathrm{F}_{25 \mathrm{~mm}}(P<0.01)$, for $\mathrm{T}_{15 \mathrm{~mm}}(P<0.001)$, and for $\mathrm{T}_{25 \mathrm{~mm}}(P<0.001)$. The minimum $\mathrm{StO}_{2}$ was significantly higher in the $\mathrm{F}_{15 \mathrm{~mm}}$ group with respect to $\mathrm{T}_{15 \mathrm{~mm}}(P<0.05)$ and to $\mathrm{T}_{25 \mathrm{~mm}}(P<0.001)$. No significant differences between $F_{25 \mathrm{~mm}}$ and $T_{15 \mathrm{~mm}}$ were found for these parameters.
Downslopes, $\Delta \mathrm{StO}_{2}$, and minimum $\mathrm{StO}_{2}$ measured in the $\mathrm{T}_{25 \mathrm{~mm}}$ group were significantly different from those measured in the other groups $(P<0.001)$.

\section{Reperfusion phase}

After release of the cuff pressure, $\mathrm{StO}_{2}$ rapidly increased to (and above) baseline $\mathrm{StO}_{2}$. In $\mathrm{F}_{15 \mathrm{~mm}}, \mathrm{~F}_{25 \mathrm{~mm}}, \mathrm{~T}_{15 \mathrm{~mm}}$, and $\mathrm{T}_{25 \mathrm{~mm}}$, $\mathrm{StO}_{2}$ rise times (that is, time from minimum $\mathrm{StO}_{2}$ to baseline $\mathrm{StO}_{2}$ ) were $0.208 \pm 0.062$ minutes, $0.198 \pm 0.050$ minutes, $0.198 \pm 0.042$ minutes, and $0.147 \pm 0.033$ minutes, respectively. None of these results were significantly different between groups (Figure 3).

In contrast to the rise times, differences between the $\mathrm{StO}_{2}$ upslopes (calculated over the same interval as the rise time) were found between groups due to differences in baselines and minima $\left(\mathrm{StO}_{2}\right.$ upslope $=\left(\mathrm{StO}_{2}\right.$ baseline $-\mathrm{StO}_{2}$ minimum)/Rise time). Upslopes were $105 \pm 34 \% /$ minute, $158 \pm 55 \% /$ minute, $226 \pm 41 \% /$ minute, and $713 \pm 101 \% /$ minute for $F_{15 \mathrm{~mm}}, F_{25 \mathrm{~mm}}, T_{15 \mathrm{~mm}}$, and $T_{25 \mathrm{~mm}}$, respectively. The upslopes in the thenar were significantly higher than the 


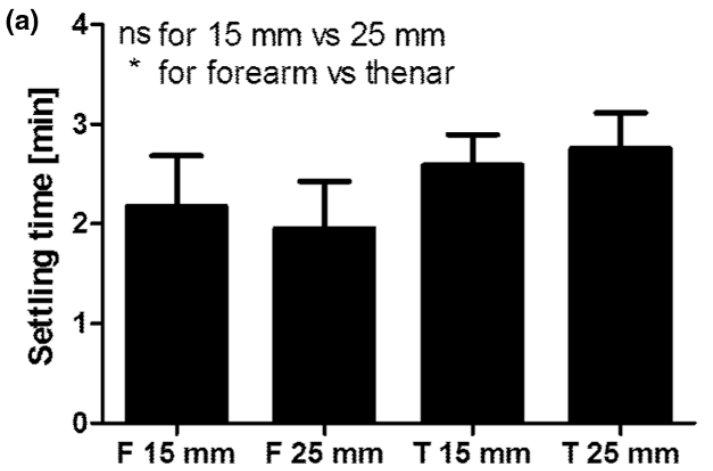

(b)

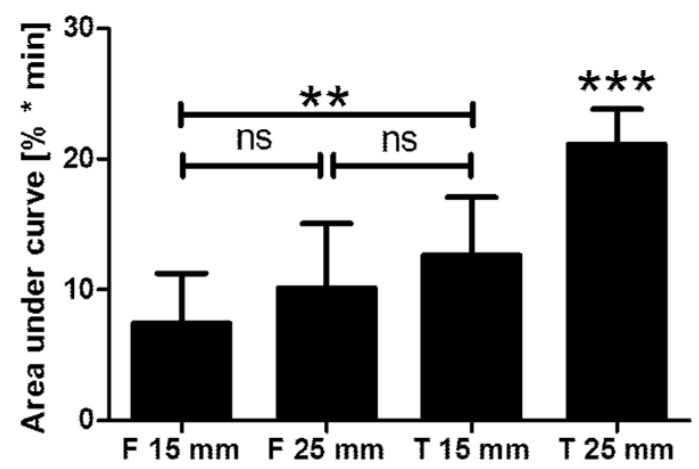

Measured settling times and the corresponding areas under the hyperemic curves. (a) Measured settling times. (b) Corresponding areas under the curves. ns $=$ not significant $(P>0.05),{ }^{\star} P<0.05,{ }^{\star \star} P<0.01,{ }^{\star \star \star} P<0.001$. F, forearm; $\mathrm{T}$, thenar.

upslopes in the forearm, and the upslopes measured with the $25 \mathrm{~mm}$ probe were significantly higher than those measured with the $15 \mathrm{~mm}$ probe (Figure 3).

Although the rise time and $\mathrm{StO}_{2}$ upslope both describe (micro)vascular reperfusion following ischemia, apparently these parameters are sensitive to different variables. Hence, where the rise time is similar for the forearm and the thenar and is independent of the applied probe, the $\mathrm{StO}_{2}$ upslope depends significantly on both the muscle and the probe type.

\section{Hyperemic phase}

Peak $\mathrm{StO}_{2}$ following release of the upper arm occlusion was $88 \pm 7 \%, 93 \pm 5 \%, 95 \pm 3 \%$, and $98 \pm 0 \%$ for $F_{15 \mathrm{~mm}}, F_{25 \mathrm{~mm}}$, $\mathrm{T}_{15 \mathrm{~mm}}$, and $\mathrm{T}_{25 \mathrm{~mm}}$, respectively, Only the peak in the $\mathrm{F}_{15 \mathrm{~mm}}$ group differed significantly from the thenar $(P<0.001$ with respect to $T_{15 \mathrm{~mm}}$ and $\left.T_{25 \mathrm{~mm}}\right)$. No significant differences were found for the $\mathrm{StO}_{2}$ overshoot (that is, peak $\mathrm{StO}_{2}-$ Baseline $\left.\mathrm{StO}_{2}\right): 6.9 \pm 3.8 \%, 8.6 \pm 3.7 \%, 8.7 \pm 3.2 \%$, and $11.3 \pm 2.7 \%$ for $F_{15 \mathrm{~mm}}, F_{25 \mathrm{~mm}}, T_{15 \mathrm{~mm}}$, and $T_{25 \mathrm{~mm}}$, respectively.

The settling time, defined as the time required for the $\mathrm{StO}_{2}$ to completely restore to baseline (Figure 4), was $2.170 \pm 0.511$ minutes, $1.950 \pm 0.475$ minutes, $2.588 \pm 0.306$ minutes, and $2.755 \pm 0.360$ minutes for $F_{15 \mathrm{~mm}}, F_{25 \mathrm{~mm}}, T_{15 \mathrm{~mm}}$, and $\mathrm{T}_{25 \mathrm{~mm}}$, respectively. No significant differences were found with respect to the probe spacing (that is, $F_{15 \mathrm{~mm}}$ versus $\mathrm{F}_{25 \mathrm{~mm}}$ $(P>0.05)$ and $\mathrm{T}_{15 \mathrm{~mm}}$ versus $\left.\mathrm{T}_{25 \mathrm{~mm}}(P>0.05)\right)$, but significant differences existed between measurement sites (that is, $\mathrm{F}_{15 \mathrm{~mm}}$ versus $\mathrm{T}_{15 \mathrm{~mm}}$ and $\mathrm{T}_{25 \mathrm{~mm}}(P<0.05)$, and $\mathrm{F}_{25 \mathrm{~mm}}$ versus $\mathrm{T}_{15 \mathrm{~mm}}$ and $\left.\mathrm{T}_{25 \mathrm{~mm}}(P<0.01)\right)$.

The AUC was $7.4 \pm 3.8 \% \cdot$ minute, $10.1 \pm 4.9 \% \cdot$ minute, $12.6 \pm 4.4 \% \cdot$ minute, and $21.2 \pm 2.7 \%$.minute for $F_{15 \mathrm{~mm}}, F_{25 \mathrm{~mm}}$, $T_{15 \mathrm{~mm}}$, and $T_{25 \mathrm{~mm}}$, respectively (Figure 4). No significant differences were found between the $15 \mathrm{~mm}$ probe and the $25 \mathrm{~mm}$ probe on the forearm. Using the $15 \mathrm{~mm}$ probe, the AUC in the thenar was significantly higher $(P<0.01)$ than in the forearm. AUCs measured in the $T_{25 \mathrm{~mm}}$ group were significantly higher than those measured in the other groups $(P<0.001)$.

\section{Correlation analysis}

To investigate the relationship between the extent of ischemia and the $\mathrm{StO}_{2}$ parameters of reperfusion and hyperemia, correlation analysis (Pearson's analysis) was performed for minimum $\mathrm{StO}_{2}$ versus reperfusion parameters $\left(\mathrm{StO}_{2}\right.$ upslope and rise time) and hyperemic parameters (peak $\mathrm{StO}_{2}, \mathrm{StO}_{2}$ overshoot, AUC, and settling time) from combined data of $F_{15 \mathrm{~mm}}, F_{25 \mathrm{~mm}}$, and $T_{15 \mathrm{~mm}} . T_{25 \mathrm{~mm}}$ data were excluded from the analysis because $\mathrm{StO}_{2}$ downslopes were not linear over the entire 3-minute period of ischemia, which would affect the consistency in the correlation analysis.

$\mathrm{StO}_{2}$ upslope correlated significantly with minimum $\mathrm{StO}_{2}$ $(r=0.81, P<0.001)$, while the rise time did not $(r=0.12$, $P>0.05)$. The peak $\mathrm{StO}_{2}(r=0.3, P<0.05), \mathrm{StO}_{2}$ overshoot $(r=0.44, P<0.001)$, and AUC $(r=0.45, P<0.01)$ exhibited a weak positive correlation with minimum $\mathrm{StO}_{2}$, whereas a correlation was absent with respect to settling time $(r=0.16$, $P>0.05)$.

To illustrate why the rise time and $\mathrm{StO}_{2}$ upslope behave differently in relation to the measurement site and probe spacing, two individual measurements are described in detail. One measurement was performed with the $15 \mathrm{~mm}$ probe on the forearm and the other with the $15 \mathrm{~mm}$ probe on the thenar, both with a baseline $\mathrm{StO}_{2}$ of $88 \%$. The $\mathrm{StO}_{2}$ downslope during ischemia was $-8 \% /$ minute in the forearm and $-16 \% /$ minute in the thenar. This resulted in different $\mathrm{StO}_{2}$ minima for the two curves: $64 \%$ in the forearm and $40 \%$ in the thenar. After release of the occlusion, both curves restored back to their baseline level in 0.233 minutes. The rise times, and thus the reperfusion dynamics, for both curves were therefore equal. The $\mathrm{StO}_{2}$ upslopes, in contrast, were very different: $103 \% /$ minute in the forearm and 206\%/minute 
in the thenar. This suggests that the $\mathrm{StO}_{2}$ upslope does not solely reflect post-ischemia reperfusion dynamics, but is also strongly influenced by the extent of $\mathrm{StO}_{2}$ decrease during ischemia.

\section{Discussion}

The primary finding of this study was that, although not apparent at baseline, the probe spacing and measurement site significantly influenced VOT-derived $\mathrm{StO}_{2}$ variables. The $\mathrm{StO}_{2}$ upslope in the reperfusion phase of the VOT was shown to depend on the minimum $\mathrm{StO}_{2}$ after 3 minutes of ischemia, while the rise time was not. Furthermore, the $\mathrm{StO}_{2}$ parameters of the hyperemic phase of the VOT were shown to significantly correlate to the minimum $\mathrm{StO}_{2}$ value after 3 minutes of ischemia.

Among the investigations employing a NIRS device identical to the ones used in the present study, some studies have used $15 \mathrm{~mm}$ probe spacing $[10,11,14]$ while others have used $25 \mathrm{~mm}$ probe spacing [9,12,16-18]. In healthy volunteers, all of these studies - including ours - have shown that baseline $\mathrm{StO}_{2}$ values were similar, independent of the applied probe. The VOT-derived $\mathrm{StO}_{2}$ variables as reported in the literature, however, varied widely between the studies using a $15 \mathrm{~mm}$ probe and the studies using a $25 \mathrm{~mm}$ probe [9-12,14,16-18]. The values obtained in the present study are comparable to those obtained in the above-referenced studies. In the present study we quantitatively compared the VOT-derived $\mathrm{StO}_{2}$ variables obtained using both probes and confirmed the hypothesis that this difference in $\mathrm{StO}_{2}$ downslopes is indeed caused by the use of different probe spacings. In the thenar, the $15 \mathrm{~mm}$ probe provided a longer time interval of linear $\mathrm{StO}_{2}$ decay during ischemia than the $25 \mathrm{~mm}$ probe, which could make the estimation of the ischemic insult and muscle metabolism inaccurate and possibly inadequate when using the $25 \mathrm{~mm}$ probe.

The potential mechanisms by which the VOT-derived $\mathrm{StO}_{2}$ traces might be different when measured by the $15 \mathrm{~mm}$ or $25 \mathrm{~mm}$ probes could be anatomy-related, physiology-related, or even technology-related. It is well established that muscle cells consume much more oxygen per unit time compared with skin and adipose tissue. Additionally, if the ischemic stimulus is more extensive in the muscle compared to the more superficial layer of (sub)dermal tissue, the reactive hyperemia is probably also of a larger extent in the deeper, muscular, layer. In this light, the probe dependence, and thus the measurement depth dependence, of the $\mathrm{StO}_{2}$ downslope and hyperemic parameters could therefore be explained by variable relative contributions of (sub)dermal tissue and muscular tissue to the NIRS signal for the different probing depths. Another option that might explain the probe dependence of the $\mathrm{StO}_{2}$ traces, however, is that the number of photons that reach the detection fiber of the NIRS probe decreases with increasing probe spacing, which, in turn, could decrease the accuracy of the $\mathrm{StO}_{2}$ calculation. This could be especially true at low microcirculatory oxygenation, as occurs during ischemia, where the optical absorbance of blood is much higher compared to at high oxygenation. This, however, is purely suggestive and no evidential data are present to support this speculation.

In the present article we provide a frame of reference for comparison of data measured in the thenar and forearm using the $15 \mathrm{~mm}$ probe and the $25 \mathrm{~mm}$ probe for a very broad spectrum of VOT-derived $\mathrm{StO}_{2}$ parameters; that is, baseline parameters, ischemic parameters, reperfusion parameters, and hyperemic parameters.

An important conceptual issue that is addressed in the present study is the difference between $\mathrm{StO}_{2}$ upslope and the rise time in the reperfusion phase of the VOT. First, it was shown that $\mathrm{StO}_{2}$ upslopes were different between the experimental groups while rise times were similar in these groups. Second, the correlation analysis performed on the minimum $\mathrm{StO}_{2}$ values after 3 minutes of ischemia versus the $\mathrm{StO}_{2}$ upslopes and rise times showed that the $\mathrm{StO}_{2}$ upslope correlated significantly with the minimum $\mathrm{StO}_{2}$ while the rise time did not. From a physiological point of view, the rise time represents the time it takes to wash out (or replace) the stagnantly deoxygenated blood in the measurement volume of the NIRS probe by oxygenated arterial blood. The $\mathrm{StO}_{2}$ upslope, on the other hand, has no single physiological meaning as it is the product of multiple variables, such as the baseline $\mathrm{StO}_{2}$, minimum $\mathrm{StO}_{2}$, and rise time. Hence, the use of rise time seems to be a more representative measure of (micro)vascular reperfusion than $\mathrm{StO}_{2}$ upslope.

Another pertinent result from the correlation analysis was the significant positive correlation between the hyperemic parameters and the minimum $\mathrm{StO}_{2}$, indicating that the extent of hyperemia is related to the extent of ischemia. This suggests that the use of a target or threshold $\mathrm{StO}_{2}$ might be more appropriate for standardization of the hyperemic phase of the VOT. After 3 minutes of ischemia, however, $\mathrm{StO}_{2}$ decreased to a minimum of $60 \%$ in the forearm and $49 \%$ in the thenar when one uses the $15 \mathrm{~mm}$ probe and to $54 \%$ in the forearm and $1 \%$ in the thenar if one uses the $25 \mathrm{~mm}$ probe. The probe type should therefore be taken into account when one uses a defined $\mathrm{StO}_{2}$ threshold value of 30 or $40 \%$. Moreover, the occlusion time might exceed 3 minutes when using the $15 \mathrm{~mm}$ probe, which could be uncomfortable for the studied subject.

Conclusively, the data from this study support the hypothesis that the NIRS measurements in combination with a VOT are measurement site-dependent and probe-dependent. The present study showed that the use of $\mathrm{StO}_{2}$ upslope is sensitive to the minimum $\mathrm{StO}_{2}$ after 3 minutes of ischemia and does not solely reflect the (micro)vascular reperfusion rate. Although the rise time seems a better measure for (micro)vascular reperfusion following ischemia, this study 
could not determine whether the use of the rise time can distinguish healthy (micro)vasculature from nonhealthy (micro)vasculature. Our study also indicated that reactive hyperemia depends on the extent of ischemic insult and supports the use of a target $\mathrm{StO}_{2}$ over the use of a fixed time of occlusion for a metabolism-independent analysis of (micro)vascular reactivity, whereby the type of probe should be taken into account. Whether the observed measurement site dependence and probe dependence is anatomy-related, physiology-related, or perhaps technology-related remains to be elucidated.

\section{Competing interests}

The NIRS devices were provided by Hutchinson Technologies.

\section{Acknowledgement}

This article is part of Critical Care Volume 13 Supplement 5: Tissue oxygenation $\left(\mathrm{StO}_{2}\right)$ in healthy volunteers and critically-ill patients. The full contents of the supplement are available online at http://ccforum. com/supplements/13/S5. Publication of the supplement has been supported with funding from Hutchinson Technology Inc.

\section{References}

1. Fink MP: Cytopathic hypoxia. Mitochondrial dysfunction as mechanism contributing to organ dysfunction in sepsis. Crit Care Clin 2001, 17:219-237.

2. Boerma EC, Mathura KR, van der Voort PH, Spronk PE, Ince C: Quantifying bedside-derived imaging of microcirculatory abnormalities in septic patients: a prospective validation study. Crit Care 2005, 9:R601-R606.

3. Ince C, Sinaasappel M: Microcirculatory oxygenation and shunting in sepsis and shock. Crit Care Med 1999, 27:1369-1377.

4. Ince C: The microcirculation is the motor of sepsis. Crit Care 2005, 9(Suppl 4):S13-S19.

5. De Backer D, Creteur J, Preiser JC, Dubois MJ, Vincent JL: Microvascular blood flow is altered in patients with sepsis. $A m$ J Respir Crit Care Med 2002, 166:98-104.

6. Astiz ME, DeGent GE, Lin RY, Rackow EC: Microvascular function and rheologic changes in hyperdynamic sepsis. Crit Care Med 1995, 23:265-271.

7. Kirschenbaum LA, Astiz ME, Rackow EC, Saha DC, Lin R: Microvascular response in patients with cardiogenic shock. Crit Care Med 2000, 28:1290-1294.

8. Trzeciak S, McCoy JV, Phillip Dellinger R, Arnold RC, Rizzuto M, Abate NL, Shapiro NI, Parrillo JE, Hollenberg SM, Microcirculatory Alterations in Resuscitation and Shock Investigators: Early increases in microcirculatory perfusion during protocoldirected resuscitation are associated with reduced multiorgan failure at $\mathbf{2 4} \mathrm{h}$ in patients with sepsis. Intensive Care Med 2008, 34:2210-2217.

9. Creteur J, Carollo T, Soldati G, Buchele G, De Backer D, Vincent $\mathrm{JL}$ : The prognostic value of muscle $\mathrm{StO}_{2}$ in septic patients. Intensive Care Med 2007, 33:1549-1556.

10. Skarda DE, Mulier KE, Myers DE, Taylor JH, Beilman GJ: Dynamic near-infrared spectroscopy measurements in patients with severe sepsis. Shock 2007, 27:348-353.

11. Gómez H, Torres A, Polanco P, Kim HK, Zenker S, Puyana JC, Pinsky MR: Use of non-invasive NIRS during a vascular occlusion test to assess dynamic tissue $\mathrm{O}_{2}$ saturation response. Intensive Care Med 2008, 34:1600-1607.

12. Pareznik R, Knezevic R, Voga G, Podbregar M: Changes in muscle tissue oxygenation during stagnant ischemia in septic patients. Intensive Care Med 2006, 32:87-92.

13. De Blasi RA, Palmisani S, Alampi D, Mercieri M, Romano R, Collin S, Pinto G: Microvascular dysfunction and skeletal muscle oxygenation assessed by phase-modulation near-infrared spectroscopy in patients with septic shock. Intensive Care Med 2005, 31:1661-1668.

14. Doerschug KC, Delsing AS, Schmidt GA, Haynes WG: Impair- ments in microvascular reactivity are related to organ failure in human sepsis. Am J Physiol Heart Circ Physiol 2007, 293: $\mathrm{H} 1065-\mathrm{H} 1071$.

15. Myers DE, Anderson LD, Seifert RP, Ortner JP, Cooper CE, Beilman GJ, Mowlem JD: Noninvasive method for measuring local hemoglobin oxygen saturation in tissue using wide gap second derivative near-infrared spectroscopy. J Biomed Opt 2005, 10:034017.

16. Strahovnik I, Podbregar M: Measurement of skeletal muscle tissue oxygenation in the critically ill. Signa Vitae 2008, 3:4350.

17. Nanas S, Gerovasili V, Dimopoulos S, Pierrakos C, Kourtidou S, Kaldara E, Sarafoglou S, Venetsanakos J, Roussos C, Nanas J, Anastasiou-Nana M: Inotropic agents improve the peripheral microcirculation of patients with end-stage chronic heart failure. J Card Fail 2008, 14:400-406.

18. Siafaka A, Angelopoulos E, Kritikos K, Poriazi M, Basios N Gerovasili V, Andreou A, Roussos C, Nanas S: Acute effects of smoking on skeletal muscle microcirculation monitored by near-infrared spectroscopy. Chest 2007, 131:1479-1485. 